upwards fixed gaze. The liver was enlarged and hypoglycemia and raised transaminases were noted. The encephalopathy and dystonic reactions were considered secondary to the anti-emetic drug in combination with liver disease most probably due to viral infection. Neurological examination was normal after 36 hours and the patient recovered completely after 10 days. The author stresses the need to consider drugs other than salicylates in the etiology or Reye syndrome and particularly the use anti-emetics (CasteelsVanDaele M. Reye syndrome or side effects of anti-emetics? Eur J Pediatr May $1991 ; \underline{150}: 456-459)$.

C OMMENT. In the 1960's several cases of toxic encephalopathy resembling Reye syndrome were reported in patients who had received the anti-emetic Tigan for vomiting. Dr. John Pepper, the Director of Toxicological Studies at Hoffman Laroche Company investigated these reports with customary thoroughness and with the aid of many consultants. No specific correlation between the use of Tigan and the toxic encephalopathy was determined. The lack of association of Reye syndrome with aspirin use has been reported from Australia (Orlowski J $\mathrm{P}$ et al. A catch in the Reye Pediatrics 1987 ; $\underline{80}: 638$. See Ped Neur Briefs Nov 1987).

\title{
HEADACHE
}

\section{CHOCOLATE IS A MIGRAINE-PROVOKING AGENT}

Patients with migraine who believe that chocolate could provoke their attacks were challenged with either chocolate or a closely matching placebo in a double-blind parallel group study at the Princess Margaret Migraine Clinic, Charing Cross Hospital, London, England. The placebo contained no cocoa butter or cocoa powder and the carob powder used in the placebo was also added to the chocolate and successfully disguised by the use of a peppermintmasking flavor. The two groups of patients, all adults, were matched for age and sex and all were drug-free for at least 48 hours prior to investigation. Chocolate ingestion (40 gram bar) was followed by a typical migraine episode in 5 out of 12 patients, while none of the 8 patients challenged with placebo had an attack $(\mathrm{P}=.051)$. The median time interval to onset of symptoms following chocolate consumption was 22 hours (range 3.5-27 hours) (Gibb C.M., Rose F.C. et al. Chocolate is a migraine provoking agent, Cephalalgia May 1991; 11:93-95).

COMMENT. In a double-blind challenge study in children referred with severe headache, 82 of 99 patients responded to an oligoantigenic diet (Egger J et al. Lancet 1983; 2:865). Relapses occurred in 74 on the introduction of one or more food stuffs, with chocolate provoking attacks in $30 \%$. Methyl-zanthine, theobromine and phenolic compounds 
including catechin are some pharmacologically active compounds found in relatively large amounts in chocolate. The relevant chemical agent responsible for initiating a migraine attack requires to be elucidated. My own clinical experience in children suggests that chocolate is a common precipitating factor in migraine susceptible patients. The effects of eliminating chocolate and caffeine containing drinks should be investigated before long-term drug treatment is considered.

\section{BLOOD MAGNESIUM LEVELS IN MIGRAINE}

Serum and erythrocyte magnesium levels were screened between attacks in patients with migraine without aura $(n=38)$ and with aura $(n=6)$ and for comparison, in a group of patients suffering from chronic tension-type headache $(n=25)$ as well as a group of control patients $(n=19)$ at the Headache Unit, Citadelle University of Liege, Liege, Belgium. Serum magnesium levels were not significantly different between the four groups of patients, whereas magnesium levels in erythrocytes were significantly reduced in the group of migraineurs without aura (1.87) compared with those with aura (2.1), patients with tension-type headache (2.03) and the controls (2.12). Normal values in the laboratory ranged from 2-2.8 $\mathrm{mmol} / \mathrm{l}$ in erythrocytes (Schoenen J. et al. Blood magnesium levels in migraine. Cephalagia May 1991; 11:97-99).

COMMENT. The correlation between magnesium levels and migraine pathophysiology is speculative. The possible importance of magnesium in migraine is suggested by the finding of reduced magnesium in white blood cells in women with premenstrual syndrome and headaches and the benefits of oral treatment with magnesium in premenstrual headaches. The authors hypothesize that the reduction in erythrocyte magnesium may be due to an abnormal regulation of intracellular magnesium in migraine patients.

\section{ESTROGENS, PROGESTINS, AND HEADACHE}

Approaches to the therapy of hormone-related headaches are reviewed from the Department of Neurology, Temple University School of Medicine and the Comprehensive Headache Center at Germantown Hospital, Philadelphia, PA, and the Reproductive Endocrine Unit, National Institute of Child Health and Human Development, NIH, Bethesda, MD. Migraine can occur before, during, or after menstruation, or at the time of ovulation. During menstruation, it is often associated with dysmenorrhea and before or during menstruation, migraine is frequently refractory to treatment. These are the times of greatest fluctuation in estrogen levels. The primary trigger of menstrual migraine appears to be the withdrawal of estrogen rather than progesterone. Changes in the sustained estrogen levels with pregnancy (increased) and menopause (decreased) can result in changes in headache frequency and intensity. Women who have migraine exclusively with their menses can be treated by the perimenstrual use of prophylactic medication (antidepressants, beta- 